\title{
Endothelium-osteoblast crosstalk
}

A new study published in Nature Medicine identifies osteoblastendothelial cell crosstalk via Slit homolog 3 protein (SLIT3) as central to bone formation. "Osteoblasts produce high levels of the axon guidance cue SLIT3," says corresponding author Matthew Greenblatt, "which increases bone formation by acting indirectly as an angiogenic factor to promote the growth of a subtype of vascular endothelium."

The bone marrow-localized $\mathrm{CD} 31^{\text {hi }}$ endomucin $(\mathrm{EMCN})^{\mathrm{hi}}$ subtype of endothelium has been implicated previously in regulating bone turnover in response to plateletderived growth factor type $\mathrm{BB}$ secreted by pre-osteoclasts. These new data, however, are the first evidence of an osteoblast-derived signal that instructs the microvasculature to initiate bone growth.

The researchers found an increase in CD $31^{\text {hi }}$ EMCN $^{\text {hi }}$ vasculature in the bones of osteoblast-specific inducible $\operatorname{Shn} 3$ (also known as Hivep3)-knockout mice, which have excess bone formation. Osteoblast cultures from $\mathrm{Shn}^{-/-}$mice were then used to make conditioned medium to screen for angiogenic factors by RNA-seq, and SLIT3 was the only major differentially expressed factor when compared with medium from wild-type osteoblast cultures.

Bone marrow endothelial progenitors treated with recombinant SLIT3 proliferated, and had increased migratory potential and tube forming activity.

By contrast, osteoblast-specific Slit3 $^{-/-}$mice had less CD $31^{\text {hi EMCN }}{ }^{\text {hi }}$ skeletal endothelium and bone mass than wild-type mice. SLIT proteins signal via the roundabout homolog (ROBO) family; the researchers identified ROBO1 as the main endothelial SLIT3 receptor.

Deletion of SLIT3 in mice with an open femoral midshaft fracture resulted in non-union, whereas deletion of Shn3 had the opposite effect, as did treatment

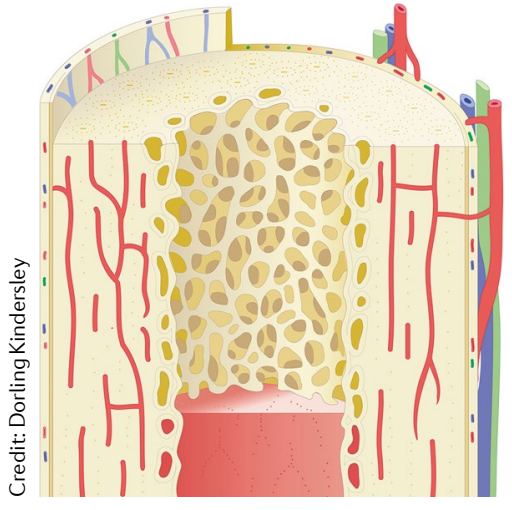

with recombinant SLIT3 delivered in a collagen sponge to the fracture site, a method designed to avoid off-target effects.

Finally the researchers also showed that recombinant SLIT3 was as effective as parathyroid hormone in therapeutic application to reverse the bone-loss recombinant SLIT3 was as effective as parathyroid hormone effects of ovariectomy, a mouse model of postmenopausal osteoporosis, hinting at future therapeutic applications for bone diseases.

Nicholas J. Bernard

ORIGINAL ARTICLE Xu, R. et al. Targeting skeletal endothelium to ameliorate bone loss. Nat. Med.

https://doi.org/10.1038/s41591-018-0020-z (2018)

\section{A cellular cascade of GM-CSF production}

\section{Granulocyte-macrophage} colony-stimulating factor (GM-CSF), an important cytokine in the development and progression of inflammatory arthritis, is produced by a variety of different cells including Thelper 17 $\left(T_{H} 17\right)$ cells, well-known contributors to arthritis pathology. "We have demonstrated that a non-T cell source of GM-CSF is indispensable for the development of autoimmune arthritis and highlighted GM-CSF-producing synovial innate lymphoid cells (ILCs) as a previously unappreciated population in inflamed joints," reports Keiji Hirota, co-corresponding author of a new study published in Immunity.

To investigate the various sources of GM-CSF and their contribution to autoimmune pathology, Hirota and colleagues used SKG mice, a model of spontaneous T cell-mediated autoimmune arthritis. As expected, GM-CSF-deficient mice were highly resistant to disease induction. Using an adoptive $T$ cell transfer system, they showed that although GM-CSF production by $T$ cells augmented chronic inflammation, this process was dispensable for arthritis initiation. Instead, GM-CSF production by radio-resistant stromal cells, including fibroblast-like synoviocytes (FLSs) and ILCs, was crucial for the development of autoimmunity. In vitro, FLSs secreted GM-CSF in response to recombinant IL-17, whereas ILCs produced GM-CSF in response to IL-2, IL-33 and Toll-like receptor 9 (TLR9) ligands. Thus, various mechanisms can contribute to the production of this pathogenic cytokine.

"GM-CSF-producing synovial ILCs were present in the inflamed joints of SKG mice and patients with rheumatoid arthritis (RA), augmenting the disease," explains Hirota. ILCs of a similar phenotype were present in the joints of healthy C57/BL6 mice, and

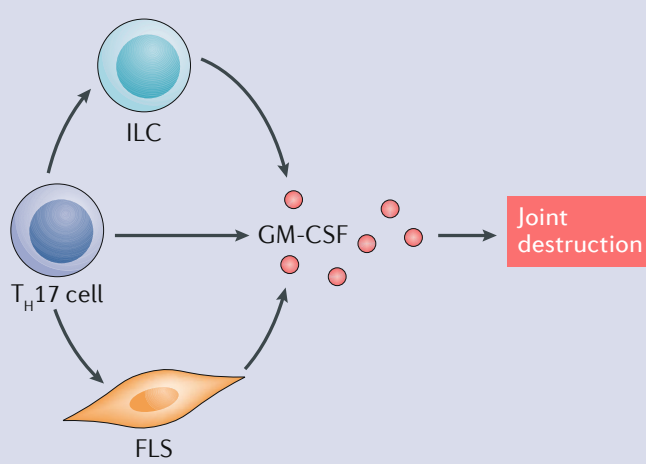

... although

GM-CSF production by $T$ cells augmented chronic inflammation, this process was dispensable for arthritis initiation g this population expanded following induction of collagen-induced arthritis (another model of RA).

"Our findings provide new insight into our understanding of the cellular sources of GM-CSF and the inflammatory cascade instigated by autoimmune $\mathrm{T}_{\mathrm{H}} 17$ cells," says co-corresponding author Shimon Sakaguchi. "Targeting this pathway might be a key therapeutic strategy for the treatment of autoimmune diseases including RA." Jessica McHugh

ORIGINAL ARTICLE Hirota, K. et al. Autoimmune Th17 Cells induced synovial stromal and innate lymphoid cell secretion of the cytokine GM-CSF to initiate and augment autoimmune. Immunity. https://doi.org/10.1016/j.immuni.2018.04.009 (2018) 